\title{
A murine model of hepatitis B-associated hepatocellular carcinoma generated by adeno-associated virus-mediated gene delivery
}

\author{
YA-HUI HUANG ${ }^{1,2}$, CHENG-CHIEH FANG ${ }^{2,3}$, KOICHI TSUNEYAMA ${ }^{4}$, HO-YUAN CHOU $^{2,5}$, \\ WEN-YU PAN ${ }^{2,6}$, YAO-MING SHIH ${ }^{2,5}$, PING-YI WU ${ }^{2}$, YIN CHEN ${ }^{2}$, \\ PATRICK S.C. LEUNG $^{7}$, M. ERIC GERSHWIN ${ }^{7}$ and MI-HUA TAO ${ }^{1,2}$
}

${ }^{1}$ Graduate Institute of Life Sciences, National Defense Medical Center; ${ }^{2}$ Institute of Biomedical Sciences, Academia Sinica; ${ }^{3}$ Department of Microbiology and Immunology, National Yang-Ming University, Taipei, Taiwan; ${ }^{4}$ Department of Diagnostic Pathology, University of Toyama, Toyama, Japan; ${ }^{5}$ Graduate Institute of Microbiology, National Taiwan University; ${ }^{6}$ Molecular Medicine Program, Taiwan International Graduate Program, Institute of Biomedical Sciences, Academia Sinica and School of Life Sciences, National Yang-Ming University, Taipei, Taiwan; ${ }^{7}$ Division of Rheumatology/Allergy and Clinical Immunology, School of Medicine, University of California, Davis, CA 95616, USA

Received June 2, 2011; Accepted July 12, 2011

DOI: 10.3892/ijo.2011.1145

\begin{abstract}
A relevant animal model is critical for investigating the pathogenic mechanisms underlying hepatitis $\mathrm{B}$ virus (HBV)-induced hepatocellular carcinoma (HCC). Mice are not naturally infected by $\mathrm{HBV}$, presumably due to the lack of HBV receptors on mouse hepatocytes. To bypass this entry step of HBV infection, we report generation of a novel HBV model in immunocompetent mice by hepatic delivery of the HBV genome using trans-splicing adeno-associated viral vectors (AAV/HBV). We confirmed production of $\mathrm{HBV}$ virions and proteins in the liver and circulation in all AAV/ $\mathrm{HBV}$-transduced mice in all four immunocompetent mouse strains tested. These mice produced antigen and antibody profiles similar to that observed in chronic HBV patients. Importantly, 12-16 months later, all 12 AAV/HBV-transduced mice developed macroscopically visible liver-tumor nodules. Ten of the twelve tumors were characterized with typical HCC features. This AAV/HBV-transduced murine HCC model provides a useful instrument for studying the pathogenesis of $\mathrm{HBV}$-associated $\mathrm{HCC}$ and the development of $\mathrm{HCC}$ therapeutic interventions.
\end{abstract}

\section{Introduction}

Hepatitis B virus (HBV) chronically infects 400 million people worldwide and leads to a high incidence of severe liver complications, including cirrhosis and hepatocellular

Correspondence to: Dr Mi-Hua Tao, 128 Academia Rd., Institute of Biomedical Sciences, Academia Sinica, Taipei, Taiwan, R.O.C.

E-mail: bmtao@ibms.sinica.edu.tw

Key words: hepatitis B virus, hepatocellular carcinoma, adenoassociated virus, mouse model carcinoma (HCC), in these patients $(1,2)$. HCC is a leading cause of death worldwide and is one of the most difficult cancers to treat $(3,4)$, only a small number of patients qualify for curative therapies. The mechanisms responsible for HCC development in chronic HBV patients are not completely understood. Evidence suggests that both the direct viral effect, such as the integration of HBV DNA into host chromosome $(5,6)$ and the transcriptional activity of $\mathrm{HBx}$ protein $(7)$, and the indirect effect of host immune-mediated inflammation and regeneration cycle $(8,9)$ might be involved in HBV-associated hepatocarcinogenesis. However, progress in understanding the pathogenic mechanisms of HBV-associated HCC has been hampered by the lack of a convenient small animal model.

Chimpanzees are susceptible to HBV infection, but only develop a mild liver inflammatory reaction (10), and their use in laboratory research is further limited because of ethical and financial considerations. Studies of HBV-related viruses in woodchucks, ground squirrels and ducks have improved our knowledge of HBV virology and the development of antiviral agents, but have not led to a better understanding of HBV immunopathology (11). Similarly, HBV transgenic mice produce infectious HBV from the chromosome-integrated viral genome, but are centrally tolerant to viral antigens and do not develop liver diseases (12). A refinement of this latter system, involving the injection of immunocompetent mice with a plasmid containing a full-length HBV genome, resulted in transient (13) or long-term (14) HBV replication in the liver, but caused only limited hepatitis. Adoptive transfer of unprimed splenocytes into HBV transgenic mice on a severe combined immunodeficiency background generated chronic hepatitis with fluctuations in alanine aminotransferase (ALT) levels (15), but the liver disease was mild and did not progress to HCC, possibly due to the lack of regeneration of $\mathrm{HBV}$-specific $\mathrm{T}$ cells in the host.

Here, we report the successful transduction and expression of HBV in the liver of immunocompetent mice using the hepatotropic adeno-associated virus serotype 8 (AAV8) vectors 

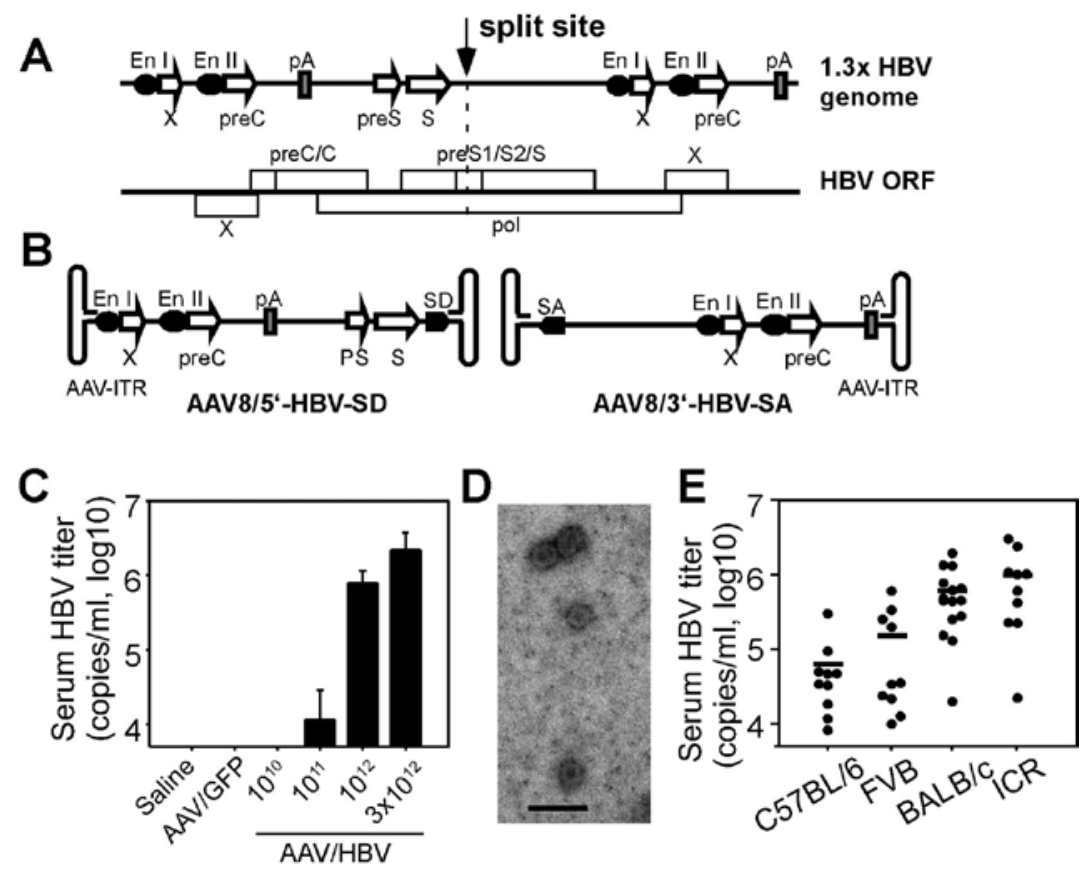

Figure 1. Co-injection of mice with trans-splicing AAV vectors produces HBV DNA and virions. (A) The 1.3x HBV genome and HBV open reading frames are shown. The split site on the HBV genome for trans-splicing AAV design is indicated by an arrow. The pre-C, pre-S, S and X promoters are indicated by open arrows. En, enhancer; pA, polyadenylation signal. (B) Schematic diagram of the trans-splicing AAV vectors. AAV/5'-HBV-SD contains the 5'-half of the 1.3x HBV genome and the splice donor (SD) sequence, while AAV/3'-HBV-SA contains the splice acceptor (SA) sequence followed by the 3'-half of the 1.3x HBV genome. (C) BALB/c mice ( $\mathrm{n}=5)$ were injected i.v. with saline, AAV/GFP ( $2 \times 10^{12} \mathrm{vg}$ per mouse), or equal doses of both AAV/5'-HBV-SD and AAV/3'-HBV-SA (between $10^{10}$ and $3 \times 10^{12} \mathrm{vg}$ of each per mouse). At one week after AAV injection, serum HBV titers were measured by real-time PCR (detection limit $5 \times 10^{3}$ copies $/ \mathrm{ml}$ ). The values are expressed as the mean \pm SD. (D) Electron microscopic analysis revealed the presence of infectious HBV Dane particles in the serum of mice receiving both AAV/5'-HBV-SD and AAV/3'-HBV-SA (10 $12 \mathrm{vg}$ of each vector per mouse). This AAV dosage was used in all subsequent experiments and is denoted as AAV/HBV transduction. Scale bar, $100 \mathrm{~nm}$. (E) Mean and individual serum HBV titers in C57BL/6 ( $\mathrm{n}=10), \mathrm{FVB}(\mathrm{n}=10), \mathrm{BALB} / \mathrm{c}(\mathrm{n}=15)$, and ICR $(\mathrm{n}=10)$ mice receiving AAV/HBV.

(16). There are several advantages of using $\mathrm{AAV}$ as a vehicle for the delivery of the HBV genome. First, the recombinant AAV vector is defective and contains a genome mostly composed of HBV DNA with only two AAV inverted terminal repeat (ITR) sequences flanking both ends (17). After binding and entry into hepatocytes (18), the AAV/HBV vectors produce only HBV but not AAV virions and proteins. Second, in transduced cells the AAV DNA genome is slowly converted to large multimers (or concatamers), mainly in a head-to-tail orientation, through ITR recombination (19). The AAV concatamer DNA, like HBV covalently closed circular DNA, is episomally maintained and serves as the template for persistent transgene expression. Third, both AAV and HBV are poorly detected by the host innate immune system (20-23), favoring their longterm persistence in target cells. We demonstrate that mice transduced with AAV/HBV vectors showed persistent HBV DNA and protein expression. Importantly, we observed HCC development in AAV/HBV-transduced mice. We suggest that this AAV/HBV-induced HCC model will be a useful tool for studying the pathogenic mechanisms of HBV-associated HCC and development of HCC therapeutic drugs.

\section{Materials and methods}

Construction and production of AAV vectors. Plasmid pHBV1.3 (24) (Fig. 1A), containing the 1.3-times overlength HBV genome (genotype D), was split at the $\mathrm{CAG} / \mathrm{G}$ site between nucleotides 1359 and 1360 (the start codon of core gene was defined as 1), and a highly conserved synthetic intron (25) inserted by PCR. The primer pairs for the 5'-HBV donor fragment (2214 bp) and 3'-HBV acceptor fragment (1976 bp) were 5'-AAAAAGCTT ATGTATTCAATCTAAGCAG-3'; 5'-TTTCTCGAGTAT TGGTCTCCTTAAACCTGTCTTGTAACCTTGATACTT ACCTGAACTGGAGCCACCAGC-3' and 5'-AAAGAA TTCCTCTTGCGTTTCTGATAGGCACCTATTGGTCTT ACTGACATCCACTTTGCCTTTCTCTCCACAGGAACA GTAAACCCTGTTCTG-3'; 5'-TTTGTCGACTACTGAA GGAAAGAAGTCAG-3'. The reverse primer for the donor HBV fragment contains the intronic donor sequence from the first intron in the human $\beta$-globulin gene, while the forward primer for the acceptor HBV fragment contains the intronic acceptor sequences from the human immunoglobulin heavy chain gene. These two PCR products were subcloned into the pAAV-MCS vector (Stratagene, La Jolla, CA), which contains the ITR of AAV serotype 2 at both ends, to generate plasmids pAAV5'-HBV-SD and pAAV-3'-HBV-SA. The pAAV-GFP plasmid expressing GFP was a gift from Dr Jin-Jer Cheng (Academia Sinica, Taipei, Taiwan). Pseudotyped AAV8 vectors carrying the 5'-HBV-SD, 3'-HBV-SA, or GFP coding sequence were generated by the triple transfection method and purified by $\mathrm{CsCl}$ sedimentation (17). The physical vector titers were assessed by quantitative PCR (26). 
Animals. All experimental procedures were reviewed and approved by the Academia Sinica IACUS which is based on Council of Agriculture Guidebook for the Care and Use of Laboratory Animals. The BALB/c, C57BL/6 and FVB mice were purchased from the National Laboratory Animal Breeding and Research Center (Taipei, Taiwan). The ICR mice were purchased from BioLASCO (Ilan, Taiwan). All animals were housed in a specific pathogen-free environment in the animal facilities of the Institute of Biomedical Sciences, Academia Sinica.

$A A V$ injections. All mice were injected i.v. at 6-8 weeks of age with the indicated titer of AAV/5'-HBV-SD, AAV/3'-HBV-SA, or both AAV/5'-HBV-SD and AAV/3'-HBV-SA (AAV/HBV). Mice injected with AAV/GFP were used as negative controls. Sera and tissue samples were collected at different times post AAV injection.

$P C R$. For quantification of the reconstituted HBV genome, serum HBV DNA was extracted using the QuickGene-810 automated nucleic acid isolation system (Fujifilm, Japan) and quantified by a sensitive hybridization probe-based real-time PCR. The PCR primer pairs for HBV DNA were 5'-CTCCA CCAATCGCCAGTC-3' which was near the end of 5' half of HBV genome and 5'-ATCCTCGAGAAGATTGACGATAAT-3' which was near the head of 3 ' half of HBV genome. The 3'-fluorescein labeled donor and 5'-Red640-labeled acceptor probes were 5'-CATGGCCTGAGGATGAGTGTTTCTCA-3' and 5'-AGGTGGAGACAGCGGGGTAGG-3' (LightCycler FastStart, Roche Diagnostics, Mannheim, Germany). Plasmid pHBV1.3 was prepared at 10 -fold dilutions $\left(1.33 \times 10^{3}-1.33 \times 10^{9}\right.$ copies $/ \mathrm{ml}$ ) to generate a standard curve in parallel PCR reactions.

Serological analysis. Sera samples were collected at different times post AAV injection. Serological markers for HBV (HBs, $\mathrm{HBe}$, anti-HBs, anti-HBe and anti-HBc) were quantified using an Elecsys Systems electrochemiluminescence kit and a Cobas e analyzer (Roche Diagnostics GmbH, Mannheim, Germany).

Electron microscopy. Particles in serum samples were concentrated on a $10 \%$ sucrose gradient by ultracentrifugation at $287,730 \mathrm{x}$ g at $4^{\circ} \mathrm{C}$ for $12 \mathrm{~h}$. The concentrated pellet was resuspended in $50 \mathrm{mM}$ Tris- $150 \mathrm{mM} \mathrm{NaCl}, \mathrm{pH} 7.4$ buffer, negatively stained with $2 \%$ uranyl acetate on a carbon-coated grid, and examined by transmission electron microscopy using a Tecnai G2 Spirit TWIN (FEI Company, Hillsboro, OR) operating at $75 \mathrm{kV}$.

Histology and immunohistochemistry. Formaldehyde-fixed and paraffin-embedded liver tissues were sectioned at $5 \mu \mathrm{m}$, mounted, heat-fixed onto glass slides, and subjected to hematoxylin-eosin $(\mathrm{H} \& \mathrm{E})$ staining for general histological inspection, and Sirius Red staining for collagen fiber analysis. For immunohistochemical staining, tissue sections were deparaffinized, soaked in target retrieval solution (TRS, $\mathrm{pH} 6.1$, Dako), and irradiated $(500 \mathrm{~W})$ in a microwave oven for $15 \mathrm{~min}$. The tissue sections were then treated with $3 \%$ hydrogen peroxide to block any endogenous peroxidase, and blocked with M.O.M. mouse immunoglobulin blocking reagent (Vector Laboratories, Burlingame, CA). The primary antibodies used were mouse anti-HBs (clone 3E7), rabbit anti$\mathrm{HBc}$, and rabbit anti-fibrinogen (all from Dako, Carpinteria, CA). The sections were then washed and incubated with the corresponding horseradish peroxidase-conjugated secondary antibodies. After thorough washing, the sections were immersed in 3,3'-diaminobenzidine (DAB, Sigma-Aldrich, St. Louis, MO) or 3-amino-9-ethylcarbazo (AEC, Sigma-Aldrich) and counterstained with hematoxylin.

Statistical analysis. All data were analyzed for significance by the Student's t-test. $\mathrm{p}<0.05$ was considered significant.

\section{Results}

Construction of trans-splicing AAV vectors. Since mouse hepatocytes are known to support HBV replication (27), the failure of $\mathrm{HBV}$ infection in mice is presumed to be due to the absence of HBV receptors on mouse hepatocytes. To bypass this entry step of HBV infection, we used the hepatotropic AAV serotype 8 vector (AAV8) (16) to introduce the HBV genome into mouse hepatocytes. To fulfill safety requirements of our institute, we employed the AAV trans-splicing technique $(25,28)$ to generate two independent AAV vectors, AAV/5'HBV-SD and AAV/3'-HBV-SA, each carrying approximately half of the HBV genome flanked by donor or acceptor splice sequences (Fig. 1A and B). We hypothesized that coadministration of these two vectors would generate functional HBV pregenomic and messenger RNAs after head-to-tail intermolecular concatamerization and productive transcription and splicing of the reconstituted HBV genome.

$H B V$ production by trans-splicing $A A V$ vectors. Male BALB/c mice (6- to 8-weeks-old) co-injected intravenously (i.v.) with equal amounts of AAV/5'-HBV-SD and AAV/3'-HBV-SA (denoted hereafter $\mathrm{AAV} / \mathrm{HBV}$ ) ranging from $10^{10}$ to $3 \times 10^{12}$ vector genomes $(\mathrm{vg})$ of each vector per mouse $(5$ mice per dose) produced HBV DNA in the serum in a dose-dependent manner (Fig. 1C). Two PCR primers, one annealing to the 5'-half HBV genome and the other to the 3'-half HBV genome, were designed to amplify only the recombined HBV genome. The PCR product from mice receiving both AAV/5'-HBV-SD and AAV/3'-HBV-SA had an expected size of 218 base pairs similar to that produced in HBV transgenic mice (Fig. 2A), and the sequence around the predicted split site $(\mathrm{CAG} / \mathrm{G})$ was identical to the expected sequence (Fig. 2B). Electron microscopy demonstrated the presence of viral particles in the serum with a size and structure similar to infectious HBV Dane particles (Fig. 1D). In contrast, mice injected with saline or control AAV8 vector expressing green fluorescent protein (AAV/GFP) at the dose of $2 \times 10^{12} \mathrm{vg}$ per mouse did not produce detectable HBV DNA in the serum. Immunohistochemical analysis of liver tissues revealed that $\mathrm{HBV}$ core $(\mathrm{HBc})$ and envelope (HBs) proteins were expressed in mice injected with the AAV/HBV vectors, but not in mice transduced with AAV/ GFP. By contrast, high GFP expression was observed in the liver of mice receiving AAV/GFP as determined by fluorescence microscopic analysis (Fig. 3). The data for the $10^{12} \mathrm{vg}$ group are shown in Fig. 3, but similar results were obtained in all groups. A dose of $10^{12} \mathrm{vg}$ was used in all subsequent 

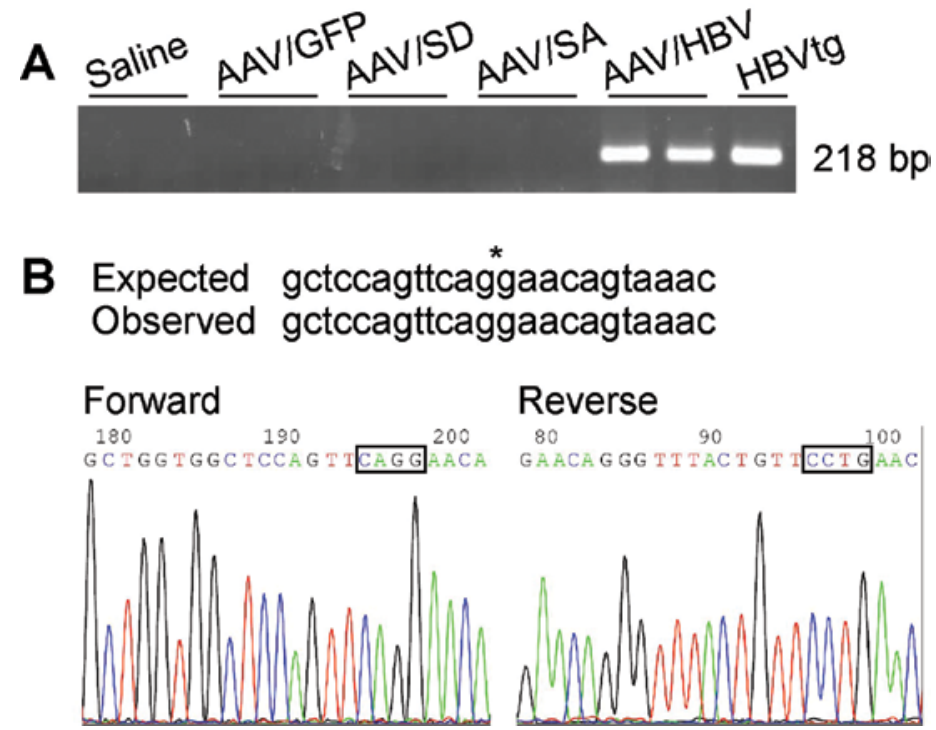

Figure 2. Evaluation of the reconstituted HBV genome after co-injection of trans-splicing AAV vectors. (A) Serum samples of AAV-transduced BALB/c mice as described in Fig. 1C were extracted and assayed for reconstituted HBV genome by PCR. The two PCR primers, one annealing to the 5'-half HBV genome and the other to the 3'-half HBV genome, were designed to amplify only the recombined HBV genome. PCR products were analyzed on $2 \%$ agarose gel. (B) PCR products of the AAV/HBV group were subcloned into pJET1.2/blunt vector and applied to sequence analysis. The data show the sequence around the split site of the HBV genome in the forward and reverse orientation. "Split site for the 1.3x HBV genome.

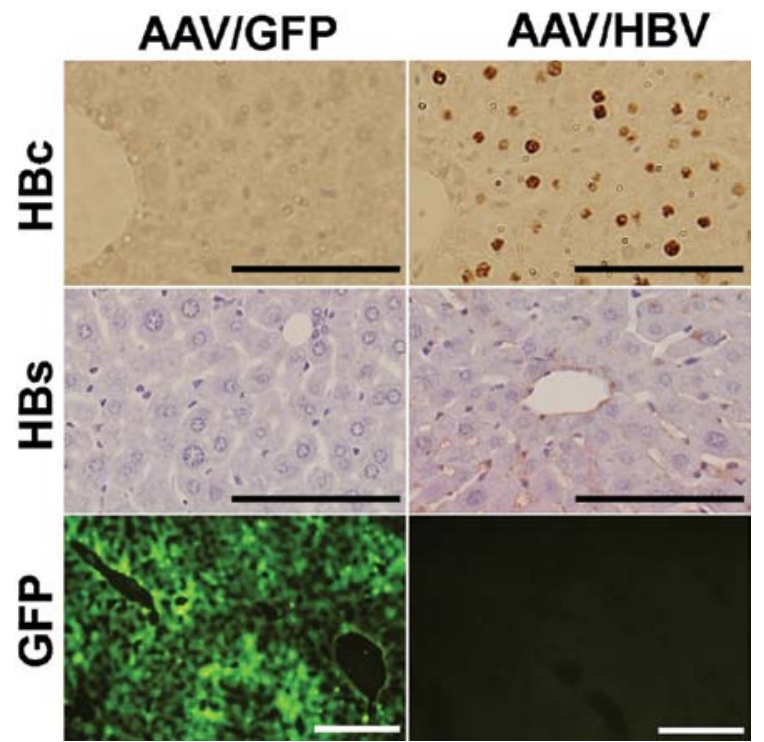

Figure 3. AAV/HBV transduction induced HBV protein expression. (A) Representative sections of the liver of mice receiving AAV/GFP or AAV/HBV, as described in Fig. 1C, stained immunohistochemically for HBc and HBs or directly examined GFP expression of liver cryosections ( $5 \mu \mathrm{m})$. Scale bar for HBc and HBs, $100 \mu \mathrm{m}$. Scale bar for GFP, $500 \mu \mathrm{m}$.

experiments. HBV protein expression was seen in the liver, but not in eight other organs examined (Fig. 4), reflecting the liver tropism of $\mathrm{HBV}$ and AAV8 vectors. Since injection of AAV/5'HBV-SD alone or AAV/3'-HBV-SA alone did not produce detectable HBV DNA in the circulation (data not shown) and the split site was located within the HBV preS1/S2/S and polymerase open reading frames (Fig. 1A), our results demonstrate that the head-to-tail intermolecular recombination of the AAV genome occurred in the co-transduced hepatocytes and led to production of fully functional HBV genomes and proteins.

To examine whether host genetic background affects HBV production by AAV-mediated HBV infection, three inbred strains [C57BL/6 $(n=10)$, FVB $(n=10)$ and BALB/c $(n=15)]$ and one outbred strain [ICR $(n=10)]$ of mice were injected i.v. with AAV/HBV. All mice used in this experiment were male. Significantly, all mice became HBV-positive 4 weeks after injection of AAV/HBV (Fig. 1E). The BALB/c and ICR mice produced higher levels of serum HBV [mean titer $6.1 \times 10^{5}$ and $1.0 \times 10^{6}$ genome copies $(\mathrm{gc})$ per $\mathrm{ml}$, respectively] than the C57BL/6 and FVB mice (mean titer $6.6 \times 10^{4}$ and $1.5 \times 10^{5} \mathrm{gc}$ per $\mathrm{ml}$, respectively).

We then examined virological features following AAV/ HBV transduction. C57BL/6 mice were given one i.v. injection of AAV/HBV $(n=4)$ or AAV/GFP $(n=4)$, then serum samples were collected 8 weeks later and tested for $\mathrm{HBV}$-specific antigens and antibodies. Significant amounts of HBs (mean titer $2541 \mathrm{IU} / \mathrm{ml}$ ) and hepatitis B e antigen (HBe, mean titer $691 \mathrm{U} /$ ml) were detected in mice transduced with AAV/HBV, but not the control AAV/GFP (Fig. 5). The AAV/HBV-transduced mice were negative for anti-HBs and anti-HBe antibodies, but positive for anti-HBc antibody (Fig. 5). This profile of HBV serological markers in AAV/HBV-transduced mice is similar to that observed in chronic hepatitis B patients $(2,29)$ and was maintained for at least 16 months following AAV/HBV transduction (Table I).

Hepatocellular carcinoma is induced in AAV/HBV-transduced mice. We then investigated whether HBV can show persistent expression in immunocompetent hosts, C57BL/6 mice were injected i.v. with AAV/HBV $(n=6)$ or AAV/GFP $(n=5)$ as described above. Throughout the 20 -week observation period, the AAV/HBV-transduced mice produced relatively stable 


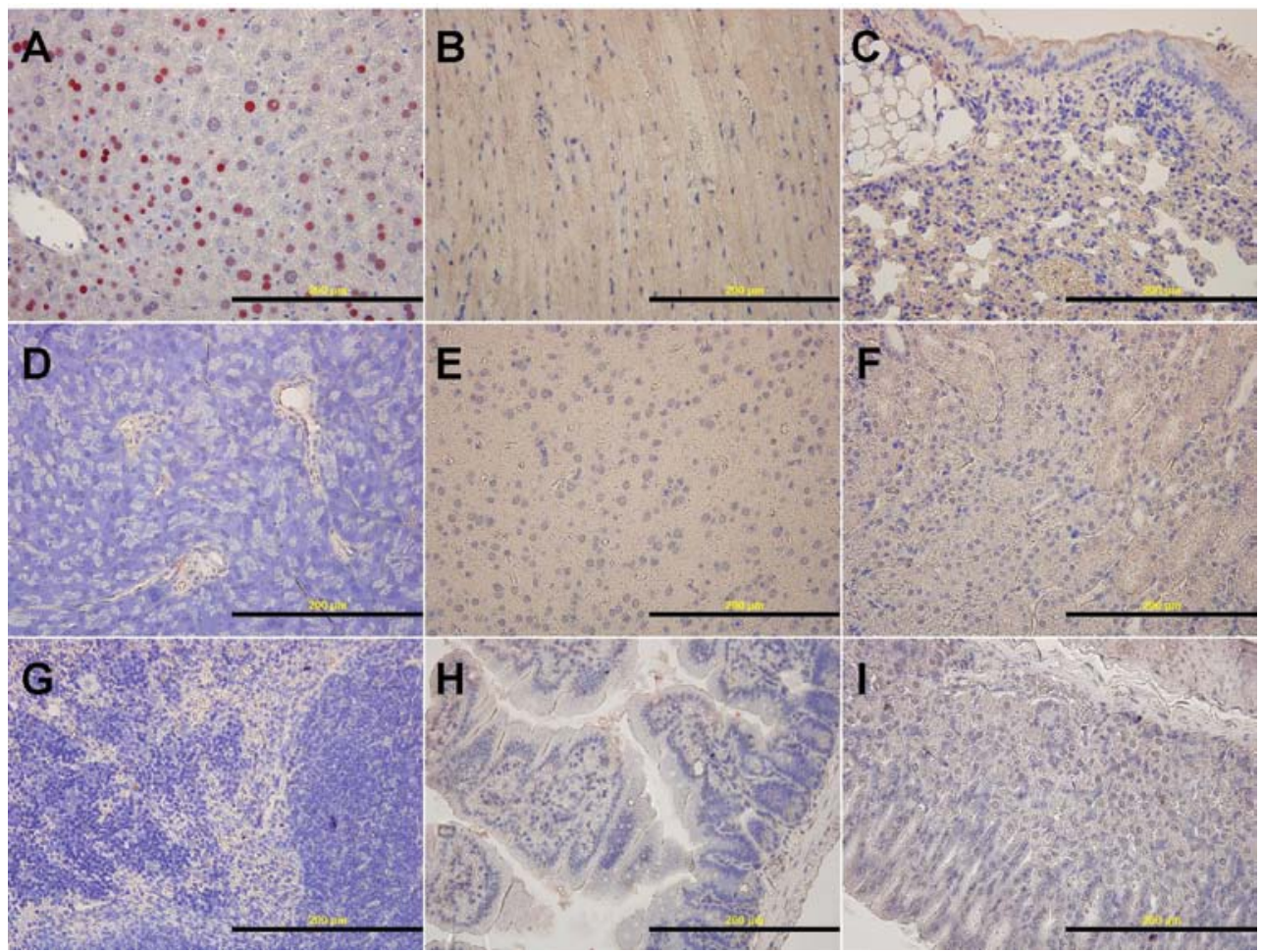

Figure 4. HBc expression in the liver (A), heart (B), lung (C), brain (D), pancreas (E), kidney (F), spleen (G), stomach (H) and intestine (I) of the AAV/HBVtransduced BALB/c mice described in Fig. 1C. Scale bar, $200 \mu \mathrm{m}$.

Table I. Biochemical and histological features of livers in AAV/HBV-transduced mice.

\begin{tabular}{|c|c|c|c|c|c|c|c|c|c|}
\hline Mouse ID & AAV & $\begin{array}{c}\text { Months } \\
\text { post-injection }\end{array}$ & $\begin{array}{l}\text { HBsAg } \\
\text { IU/ml }\end{array}$ & $\begin{array}{c}\mathrm{HBeAg} \\
\mathrm{U} / \mathrm{ml}\end{array}$ & $\begin{array}{c}\text { ALT } \\
\mathrm{U} / 1\end{array}$ & $\begin{array}{l}\text { Liver } \\
\text { tumors }\end{array}$ & $\begin{array}{l}\text { No. of visible } \\
\text { tumors }\end{array}$ & $\begin{array}{l}\text { Largest } \\
\text { tumor }(\mathrm{mm})\end{array}$ & $\begin{array}{c}\text { Tumor } \\
\text { histology }\end{array}$ \\
\hline IF1294 & $\mathrm{AAV} / \mathrm{HBV}$ & 12 & $744(+)$ & $421(+)$ & 146 & + & 1 & 16 & $\mathrm{HCC}$ \\
\hline IF1121 & $\mathrm{AAV} / \mathrm{HBV}$ & 13 & $3324(+)$ & ND & 225 & + & 2 & 22 & $\mathrm{HCC}$ \\
\hline IF1123 & $\mathrm{AAV} / \mathrm{HBV}$ & 13 & $3617(+)$ & ND & 215 & + & TMTC & 17 & $\mathrm{HCC}$ \\
\hline IF1181 & $\mathrm{AAV} / \mathrm{HBV}$ & 14 & $4432(+)$ & $66(+)$ & 271 & + & TMTC & 17 & $\mathrm{HCC}$ \\
\hline IF1182 & $\mathrm{AAV} / \mathrm{HBV}$ & 14 & $770(+)$ & $623(+)$ & 103 & + & 3 & 4 & $\begin{array}{c}\text { Dysplastic } \\
\text { nodules }\end{array}$ \\
\hline IF1252 & $\mathrm{AAV} / \mathrm{HBV}$ & 16 & $1964(+)$ & $202(+)$ & 345 & + & TMTC & 16 & $\mathrm{HCC}$ \\
\hline IF1253 & $\mathrm{AAV} / \mathrm{HBV}$ & 16 & $1662(+)$ & $37(+)$ & 266 & + & TMTC & 23 & $\mathrm{HCC}$ \\
\hline IF1254 & $\mathrm{AAV} / \mathrm{HBV}$ & 16 & $4766(+)$ & $32(+)$ & 369 & + & TMTC & 23 & $\mathrm{HCC}$ \\
\hline IF1258 & $\mathrm{AAV} / \mathrm{HBV}$ & 16 & $2912(+)$ & $21(+)$ & 370 & + & TMTC & 20 & $\mathrm{HCC}$ \\
\hline IF1259 & $\mathrm{AAV} / \mathrm{HBV}$ & 16 & $2753(+)$ & $175(+)$ & 350 & + & 2 & 23 & $\mathrm{HCC}$ \\
\hline IF1260 & $\mathrm{AAV} / \mathrm{HBV}$ & 16 & $2318(+)$ & $54(+)$ & 76 & + & ND & ND & $\begin{array}{c}\text { Dysplastic } \\
\text { nodules }\end{array}$ \\
\hline IF1261 & $\mathrm{AAV} / \mathrm{HBV}$ & 16 & $1928(+)$ & $76(+)$ & 443 & + & TMTC & ND & $\mathrm{HCC}$ \\
\hline IF1295 & AAV/GFP & 12 & - & - & 24 & - & - & - & - \\
\hline IF1737 & AAV/GFP & 16 & - & - & 70 & - & - & - & - \\
\hline IF1738 & AAV/GFP & 16 & - & - & 48 & - & - & - & - \\
\hline IF1739 & AAV/GFP & 16 & - & - & 82 & - & - & - & - \\
\hline IF1740 & AAV/GFP & 16 & - & - & 74 & - & - & - & - \\
\hline
\end{tabular}

Abbreviations: ND, not determined; TMTC, too many to count; (-), undetectable. 


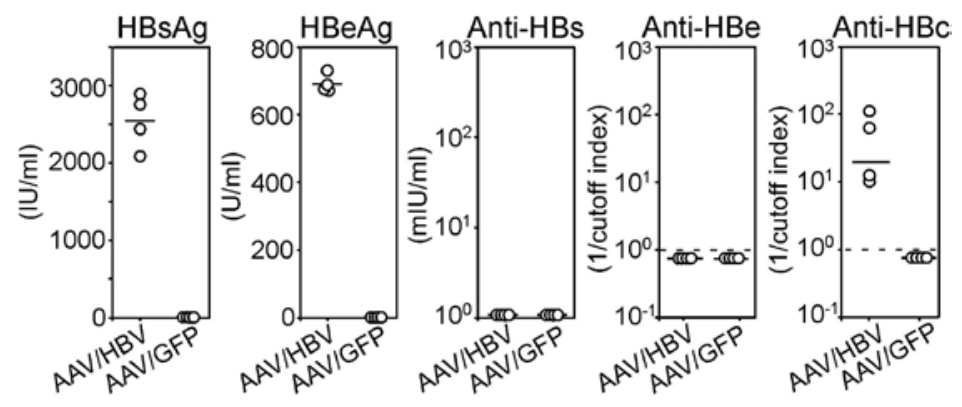

Figure 5. HBV serological responses induced by AAV/HBV transduction. C57BL/6 mice (H2 ${ }^{\mathrm{b}}$ haplotype) were injected i.v. with AAV/HBV or AAV/GFP as described above. Serum samples ( $\mathrm{n}=4$ of each group) were collected 8 weeks post AAV injection and the amounts of HBV proteins and anti-HBV antibodies measured by ELISA.

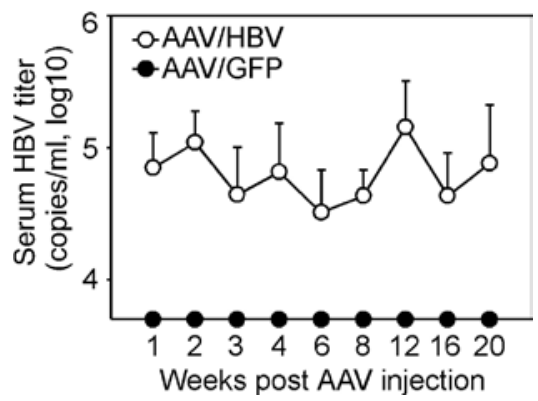

Figure 6. Kinetics of serum HBV titers after AAV/HBV transduction. C57BL/6 mice were injected with AAV/HBV $(n=6)$ or AAV/GFP $(n=5)$ as described above, then average serum HBV titers (mean \pm SD) were measured over time.

levels of serum $\mathrm{HBV}$, with a mean titer between $3 \times 10^{4}-2 \times 10^{5} \mathrm{gc}$ per ml (Fig. 6).

To investigate whether persistent HBV expression in immunocompetent mice led to development of HCC, C57BL/6 mice $(n=12)$ were injected i.v. with AAV/HBV and their livers were removed at 12-16 months after injection for macroscopic and histopathological analysis. All the AAV/HBV-transduced mice (12 of 12, 100\%) developed macroscopically visible liver tumor nodules between 12 and 16 months after AAV/HBV transduction (Table I). Fig. 7A shows an example of the multiple well-vascularized tumors (mouse IF1123, 13 months after AAV/ HBV transduction). Microscopic examination revealed that these tumors had a distinctive nodule-in-nodule appearance (Fig. 7B), in which a poorly or moderately differentiated HCC, characterized by a pseudoglandular to thick trabecular pattern (T1 region, Fig. 7C), developed within a well differentiated HCC (T2 region). Other large tumors ( $>10 \mathrm{~mm}$ in diameter) were also diagnosed as moderately to well differentiated HCC, characterized by a pseudoglandular pattern (Fig. 7D), increased cellularity and nuclear atypia (Fig. 7E), the absence of portal tracts (Fig. 7F), and occasional portal invasion by atypical hepatocytes (Fig. 7G). Round amphophilic cytoplasmic inclusions were occasionally observed in the tumor areas (Fig. 7H) and showed a strong positive immunoreaction for fibrinogen (Fig. 7H, bottom inset), similar to the structure of the pale bodies found in some human HCCs $(30,31)$. Portal and parenchymal inflammatory infiltrates with focal necrosis of multiple hepatocytes were seen through the adjacent non-tumor liver tissue (Fig. 8A and B). Pronounced steatosis was a common feature in the non-tumor region (Fig. 8C) which occasionally displayed mild to moderate liver fibrosis (Fig. 8D). In contrast, none of the AAV/GFP-transduced C57BL/6 mice $(0$ of $5,0 \%)$ developed detectable liver nodules over the 12- to 16-month observation period (Table I), and their livers showed no apparent histological changes (data not shown). Most of these AAV/GFP-transduced mice had serum ALT levels within the normal range, with an average titer of $60 \pm 24 \mathrm{U} / 1$, while ALT levels in the AAV/HBV-transduced mice of a similar age and bearing $\mathrm{HCC}$ were significantly elevated, with an average titer of $265 \pm 93 \mathrm{U} / 1$ (Table I).

\section{Discussion}

HCC is the third leading cause of cancer mortality worldwide, and chronic HBV infection is one of the major risk factors for development of this cancer $(1,2)$. The molecular mechanisms by which HBV infection leads to hepatocarcinogenesis are not completely understood, but evidence suggests that development of HCC may be related to the direct effect of the transcriptional activity of HBx protein (7) or HBV DNA integration $(5,6)$, as well as an indirect effect through immune-mediated hepatic inflammation, injury and regeneration $(8,9)$. It is also possible that a combination of viral and host factors are required for HCC development in chronic HBV patients. Therefore, an animal model that can recapitulate both viral and host features in an immunocompetent background will be a valuable tool to study the pathological aspects of HBV-associated HCC. In transgenic mouse studies, overexpression and accumulation of HBV large envelope proteins (32) or X proteins (33) led to development of HCC. However, the whole HBV genome transgenic mice, producing various levels of viral proteins and virions, did not manifest pathological changes or liver tumor development in several previous studies $(12,15,34)$. One disadvantage of using transgenic animals is their central tolerance to $\operatorname{HBV}(35,36)$, which might partly explain the lack of liver injury and of HCC development in whole HBV genome transgenic mice, since adoptive transfer of HBV-specific T cells induces a high incidence of HCC in these transgenic mice (37).

To generate a HBV animal model in immunocompetent mice, we used two trans-splicing AAV8 vectors, AAV/5'-HBV-SD and AAV/3'-HBV-SA, to deliver the entire HBV genome into mouse hepatocytes. Our results show that mice co-injected with both donor and acceptor AAV vectors (AAV/HBV) produced HBV DNA (Fig. 1C and E) and HBV proteins, 


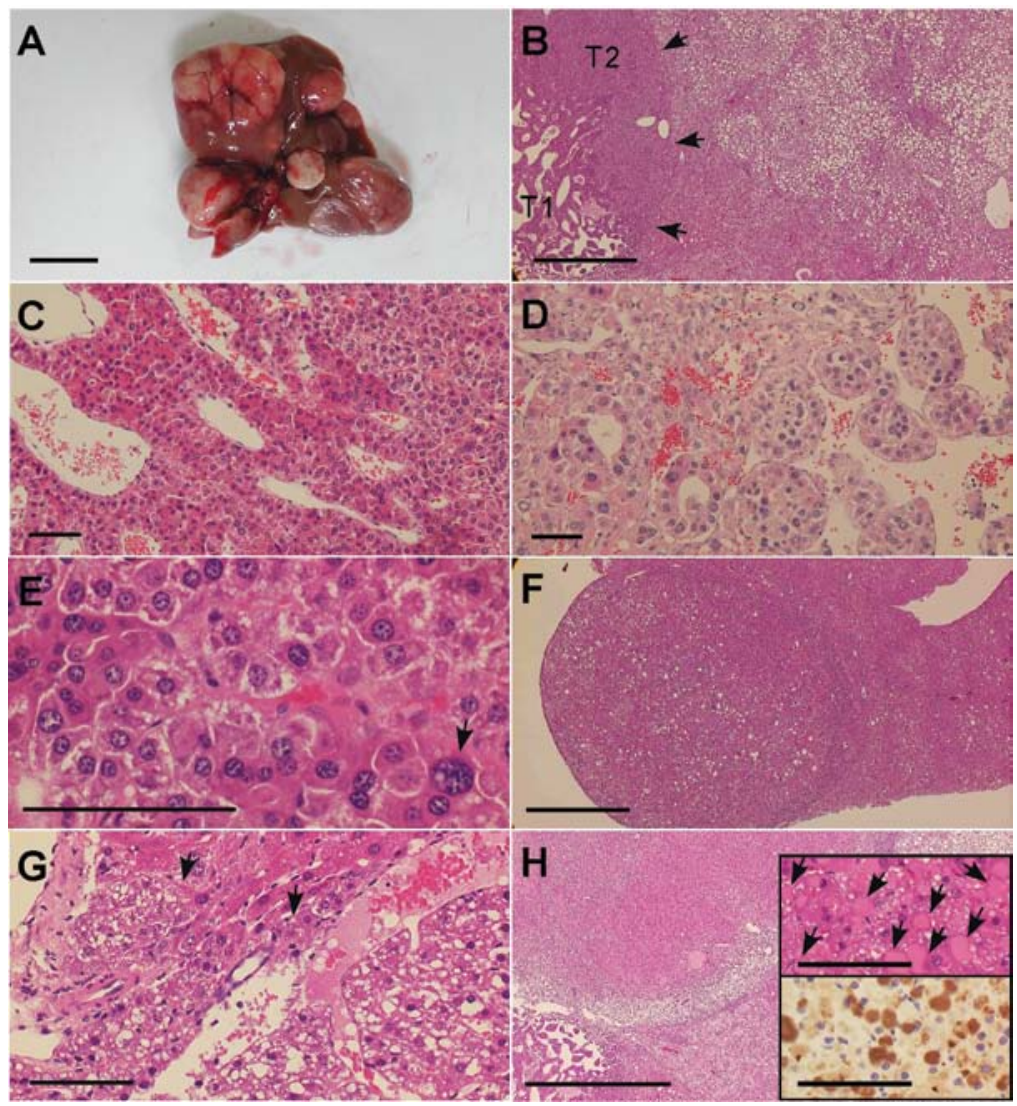

Figure 7. Mice transduced with AAV/HBV develop HCC. Wild-type C57BL/6 mice were transduced with AAV/HBV and sacrificed 12-16 months later (Table I). The liver of each mouse was photographed, then prepared as paraffin sections for histological analysis. (A-C) Gross appearance (A) and histological features (B and C) of liver tumors from one representative mouse (IF1123, 13 months after HBV transduction). The tumors displayed a distinctive nodule-innodule appearance (B), in which a moderately to poorly differentiated HCC (T1) with a thick trabecular pattern (C) develops within a well-differentiated HCC (T2). The arrows indicate the border of the tumor. (D-H) Other histological features of the liver lesions, including a pseudoglandular pattern of hepatic plates (D), increased cellularity and nuclear atypia (E, arrow), absence of portal tracks in the tumor lesions (F), portal invasion by atypical hepatocytes (G, arrows), and intra-cytoplasmic pale bodies $(\mathrm{H})$. In $\mathrm{H}$, the insets are higher magnification showing $\mathrm{H} \& \mathrm{E}$ staining (top) and fibrinogen immunostaining of the pale bodies (bottom). Scale bar, $1 \mathrm{~cm}$ (A). Scale bars, $1 \mathrm{~mm}$ (B, F and H). Scale bars, $100 \mu \mathrm{m}$ (C-E, G, inset H).

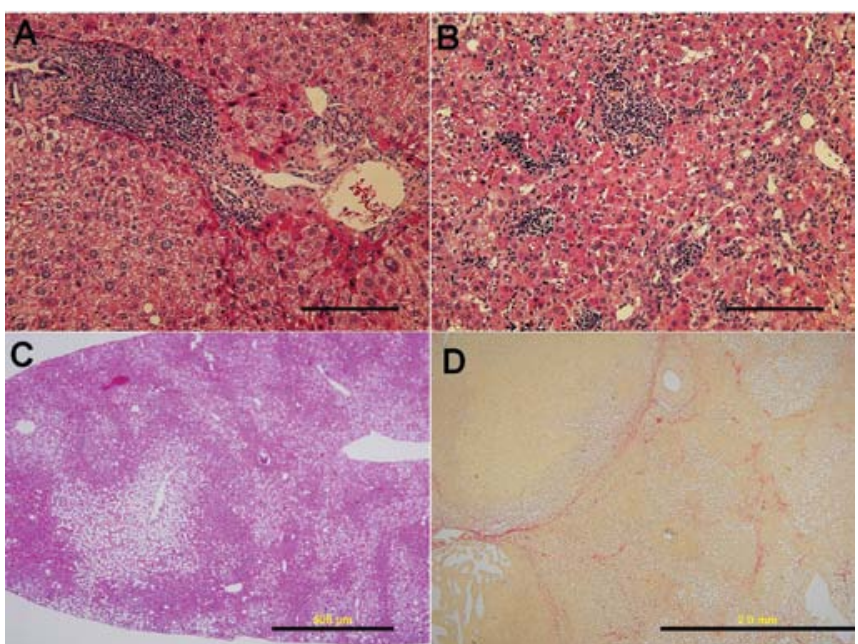

Figure 8. Histological changes in the adjacent non-tumor liver tissues in AAV/HBV-transduced mice. (A-C) H\&E staining of the non-tumor liver tissues from one representative AAV/HBV-transduced mouse with $\mathrm{HCC}$. The sections show portal (A) and parenchymal (B) mononuclear cell infiltration with focal necrosis and acidophilic bodies scattered across the sections. Pronounced steatosis (C) was commonly observed. (D) Sirius red staining reveals bridging fibrosis in the parenchyma and surrounding the tumor nodules. Scale bar, $200 \mu \mathrm{m}$ (A-B). Scale bar, $500 \mu \mathrm{m}$ (C). Scale bar, $2 \mathrm{~mm}$ (D). including secreted HBs and $\mathrm{HBe}$ in the circulation (Fig. 5) and cellular HBc and HBs in hepatocytes (Fig. 3). The DNA and protein expression patterns are similar to chronic human HBV infection (2). PCR analysis and sequencing confirmed that the HBV DNA found in AAV/HBV mice had the same sequence as that of wild-type HBV (Fig. 2), demonstrating the existence of properly oriented AAV/HBV genomes, which produced functional HBV RNAs as templates for production of HBV DNA genome and proteins.

Significantly, at between 12 and 16 months after AAV/ HBV transduction, all mice developed liver tumor nodules, manifesting pathological features of either dysplasia (17\%) or HCC (83\%) (Fig. 7 and Table I). The adjacent non-tumor liver tissue was also histologically abnormal, frequently displaying inflammatory infiltrates, steatosis, and focal necrosis (Fig. 8). Mild to moderate liver fibrosis was also occasionally observed. In contrast, none of the AAV/GFP-transduced mice on the same C57BL/6 background developed tumor nodules in the liver (Table I), which remained histologically normal on microscopic analysis.

There are several potential mechanisms which might be involved in HCC development in our AAV/HBV-transduced mice. First, the abundant HBV proteins, persistently expressed 
in AAV/HBV-transduced mice, might have a direct hepatocarcinogenic effect as that reported in human HCC patients and in transgenic mouse studies $(32,33,38,39)$. Second, as natural HBV infection in humans and chimpanzees $(2,40)$, the host immunemediated inflammation may be another factor responsible for HCC development since our AAV/HBV model is also established in the immunocompetent host. Third, AAV-mediated HBV DNA integration into chromosome of hepatocytes may also contribute to $\mathrm{HCC}$ development. AAV vectors have been found to integrate into host cell chromosomes in a non-specific manner, favoring transcriptionally active genes and DNA breakage sites $(41,42)$. However, our results (Table I) and those from other studies (43-45), including a large-scale study of 695 mice and sequencing of over 1000 integration sites, did not show any increased risk of developing liver tumors or other tumor types in AAV-treated mice. However, there also have been reports that AAV-mediated gene therapy did increase the risk of formation of liver tumors $(46,47)$ and that this is associated with AAV insertion and overexpression of the Rian gene, containing multiple small nucleolar RNAs, and of the Mirg gene, containing multiple microRNAs. We did not detect increased expression of the Rian and Mirg genes in AAV/ HBV-induced tumors (unpublished results), arguing against a role of these two genes in hepatocarcinogenesis in our HCC model. However, we can not formally rule out the possibility that the inserted HBV gene controlling elements (HBV enhancers and promoters), which are present in our model but not in AAV vectors of other studies, might play a role in liver carcinogenesis of our AAV/HBV-induced HCC model.

Compared to HCC models established in genetically engineered mice involving either overexpression of oncogenic proteins or germline disruption of tumor suppressors $(48,49)$, our AAV/HBV-transduced HCC model has the advantages of easy manipulation and recapitulation of both the viral and immune effectors known to be critical in the development of human HBV-associated HCC. This AAV/HBV murine HCC model therefore provides a useful tool for studying the pathogenic mechanisms responsible for $\mathrm{HBV}$-associated $\mathrm{HCC}$ and the development of HCC therapeutic drugs.

\section{Acknowledgements}

We thank J.J. Chen (Institute of Biomedical Sciences, Academia Sinica) for the gift of pAAV/GFP plasmid and S.R. Roffler (Institute of Biomedical Sciences, Academia Sinica) for discussions. M.-H.T. was supported by Academia Sinica Summit Program and National Research Program for Genomic Medicine grant NSC 98-3112-B-001-030 and NSC 99-3112-B-001-013.

\section{References}

1. El-Serag HB and Rudolph KL: Hepatocellular carcinoma: epidemiology and molecular carcinogenesis. Gastroenterology 132 2557-2576, 2007.

2. Ganem D and Prince AM: Hepatitis B virus infection-natural history and clinical consequences. N Engl J Med 350: 1118-1129, 2004.

3. Avila MA, Berasain C, Sangro B and Prieto J: New therapies for hepatocellular carcinoma. Oncogene 25: 3866-3884, 2006.

4. Llovet JM and Bruix J: Molecular targeted therapies in hepatocellular carcinoma. Hepatology 48: 1312-1327, 2008.
5. Tokino T, Tamura H, Hori $\mathrm{N}$ and Matsubara K: Chromosome deletions associated with hepatitis B virus integration. Virology 185: 879-882, 1991.

6. Murakami Y, Saigo K, Takashima H, et al: Large scaled analysis of hepatitis B virus (HBV) DNA integration in HBV related hepatocellular carcinomas. Gut 54: 1162-1168, 2005.

7. Muroyama R, Kato N, Yoshida H, et al: Nucleotide change of codon 38 in the $\mathrm{X}$ gene of hepatitis $\mathrm{B}$ virus genotype $\mathrm{C}$ is associated with an increased risk of hepatocellular carcinoma. J Hepatol 45: 805-812, 2006.

8. Ferrari C, Missale G, Boni C and Urbani S: Immunopathogenesis of hepatitis B. J Hepatol 39 (Suppl 1): S36-S42, 2003.

9. Guidotti LG and Chisari FV: Immunobiology and pathogenesis of viral hepatitis. Annu Rev Pathol 1: 23-61,2006.

10. Shouval D, Chakraborty PR, Ruiz-Opazo N, et al: Chronic hepatitis in chimpanzee carriers of hepatitis B virus: morphologic, immunologic, and viral DNA studies. Proc Natl Acad Sci USA 77: 6147-6151, 1980.

11. Dandri M, Volz TK, Lutgehetmann M and Petersen J: Animal models for the study of HBV replication and its variants. J Clin Virol 34 (Suppl 1): S54-S62, 2005.

12. Guidotti LG, Matzke B, Schaller H and Chisari FV: High-level hepatitis B virus replication in transgenic mice. J Virol 69: 6158-6169, 1995

13. Yang PL, Althage A, Chung J and Chisari FV: Hydrodynamic injection of viral DNA: a mouse model of acute hepatitis B virus infection. Proc Natl Acad Sci USA 99: 13825-13830, 2002.

14. Huang LR, Wu HL, Chen PJ and Chen DS: An immunocompetent mouse model for the tolerance of human chronic hepatitis B virus infection. Proc Natl Acad Sci USA 103: 17862-17867, 2006.

15. Larkin J, Clayton M, Sun B, et al: Hepatitis B virus transgenic mouse model of chronic liver disease. Nat Med 5: 907-912, 1999.

16. Gao GP, Alvira MR, Wang L, Calcedo R, Johnston J and Wilson JM: Novel adeno-associated viruses from rhesus monkeys as vectors for human gene therapy. Proc Natl Acad Sci USA 99: 11854-11859, 2002.

17. Xiao X, Li J and Samulski RJ: Production of high-titer recombinant adeno-associated virus vectors in the absence of helper adenovirus. J Virol 72: 2224-2232, 1998.

18. Akache B, Grimm D, Pandey K, Yant SR, Xu H and Kay MA: The 37/67-kilodalton laminin receptor is a receptor for adenoassociated virus serotypes 8, 2, 3, and 9. J Virol 80: 9831-9836, 2006.

19. Duan D, Sharma P, Yang J, et al: Circular intermediates of recombinant adeno-associated virus have defined structural characteristics responsible for long-term episomal persistence in muscle tissue. J Virol 72: 8568-8577, 1998.

20. McCaffrey AP, Fawcett P, Nakai H, et al: The host response to adenovirus, helper-dependent adenovirus, and adeno-associated virus in mouse liver. Mol Ther 16: 931-941, 2008.

21. Nayak S and Herzog RW: Progress and prospects: immune responses to viral vectors. Gene Ther 17: 295-304, 2010.

22. Wieland S, Thimme R, Purcell RH and Chisari FV: Genomic analysis of the host response to hepatitis B virus infection. Proc Natl Acad Sci USA 101: 6669-6674, 2004.

23. Dunn C, Peppa D, Khanna P, et al: Temporal analysis of early immune responses in patients with acute hepatitis B virus infection. Gastroenterology 137: 1289-1300, 2009.

24. Chou YC, Jeng KS, Chen ML, et al: Evaluation of transcriptional efficiency of hepatitis B virus covalently closed circular DNA by reverse transcription-PCR combined with the restriction enzyme digestion method. J Virol 79: 1813-1823, 2005.

25. Lai Y, Yue Y, Liu M and Duan D: Synthetic intron improves transduction efficiency of trans-splicing adeno-associated viral vectors. Hum Gene Ther 17: 1036-1042, 2006.

26. Chen CC,Sun CP,Ma HI, et al: Comparative study of anti-hepatitis B virus RNA interference by double-stranded adeno-associated virus serotypes 7, 8, and 9. Mol Ther 17: 352-359, 2009.

27. Chen $\mathrm{SH}, \mathrm{Hu} \mathrm{CP}$ and Chang CM: Hepatitis B virus replication in well differentiated mouse hepatocyte cell lines immortalized by plasmid DNA. Cancer Res 52: 1329-1335, 1992.

28. Lai Y, Yue Y, Liu M, et al: Efficient in vivo gene expression by trans-splicing adeno-associated viral vectors. Nat Biotechnol 23: 1435-1439, 2005.

29. Rehermann B and Nascimbeni M: Immunology of hepatitis $B$ virus and hepatitis $C$ virus infection. Nat Rev Immunol 5: 215-229, 2005.

30. Moon WS, Yu HC, Chung MJ, Kang MJ and Lee DG: Pale bodies in hepatocellular carcinoma. J Korean Med Sci 15: 516-520, 2000 . 
31. Nakashima O, Sugihara S, Eguchi A, Taguchi J, Watanabe J and Kojiro M: Pathomorphologic study of pale bodies in hepatocellular carcinoma. Acta Pathol Jpn 42: 414-418, 1992.

32. Chisari FV, Klopchin K, Moriyama T, et al: Molecular pathogenesis of hepatocellular carcinoma in hepatitis B virus transgenic mice. Cell 59: 1145-1156, 1989.

33. Kim CM, Koike K, Saito I, Miyamura T and Jay G: HBx gene of hepatitis B virus induces liver cancer in transgenic mice. Nature 351: 317-320, 1991.

34. Zheng Y, Chen WL, Louie SG, Yen TS and Ou JH: Hepatitis $\mathrm{B}$ virus promotes hepatocarcinogenesis in transgenic mice. Hepatology 45: 16-21, 2007.

35. Sette AD, Oseroff C, Sidney J, et al: Overcoming T cell tolerance to the hepatitis B virus surface antigen in hepatitis B virustransgenic mice. J Immunol 166: 1389-1397, 2001

36. Shimizu Y, Guidotti LG, Fowler P and Chisari FV: Dendritic cell immunization breaks cytotoxic $\mathrm{T}$ lymphocyte tolerance in hepatitis B virus transgenic mice. J Immunol 161: 4520-4529, 1998.

37. Nakamoto Y, Guidotti LG, Kuhlen CV, Fowler P and Chisari FV: Immune pathogenesis of hepatocellular carcinoma. J Exp Med 188: 341-350, 1998.

38. Chen CJ, Yang HI, Su J, et al: Risk of hepatocellular carcinoma across a biological gradient of serum hepatitis B virus DNA level. JAMA 295: 65-73, 2006.

39. Azam F and Koulaouzidis A: Hepatitis B virus and hepatocarcinogenesis. Ann Hepatol 7: 125-129, 2008.

40. Chisari FV and Ferrari C: Hepatitis B virus immunopathogenesis. Annu Rev Immunol 13: 29-60, 1995.
41. Nakai H, Montini E, Fuess S, Storm TA, Grompe M and Kay MA: AAV serotype 2 vectors preferentially integrate into active genes in mice. Nat Genet 34: 297-302, 2003.

42. Miller DG, Petek LM and Russell DW: Adeno-associated virus vectors integrate at chromosome breakage sites. Nat Genet 36: 767-773, 2004.

43. Bell P, Moscioni AD, McCarter RJ, et al: Analysis of tumors arising in male $\mathrm{B} 6 \mathrm{C} 3 \mathrm{~F} 1$ mice with and without $\mathrm{AAV}$ vector delivery to liver. Mol Ther 14: 34-44, 2006.

44. Bell P, Wang L, Lebherz C, et al: No evidence for tumorigenesis of AAV vectors in a large-scale study in mice. Mol Ther 12: 299-306, 2005

45. Li H, Malani N, Hamilton SR, et al: Assessing the potential for AAV vector genotoxicity in a murine model. Blood 117: 3311-3319, 2011.

46. Donsante A, Miller DG, Li Y, et al: AAV vector integration sites in mouse hepatocellular carcinoma. Science 317: 477, 2007.

47. Donsante A, Vogler C, Muzyczka N, et al: Observed incidence of tumorigenesis in long-term rodent studies of rAAV vectors. Gene Ther 8: 1343-1346, 2001.

48. Newell P, Villanueva A, Friedman SL, Koike K and Llovet JM: Experimental models of hepatocellular carcinoma. J Hepatol 48: 858-879, 2008

49. Keng VW, Villanueva A, Chiang DY, et al: A conditional transposon-based insertional mutagenesis screen for genes associated with mouse hepatocellular carcinoma. Nat Biotechnol 27: 264-274, 2009. 